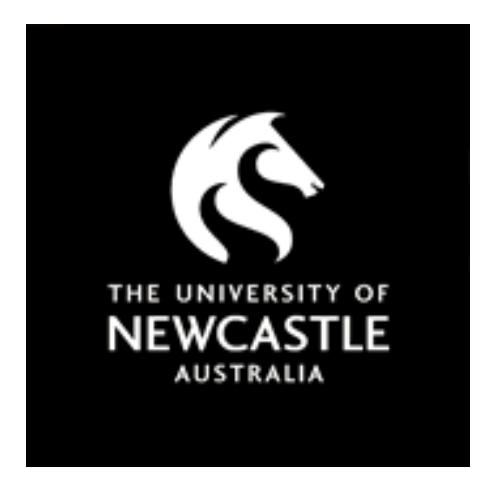

NOVA

University of Newcastle Research Online

nova.newcastle.edu.au

Ahmed, Osman H.; Lee, Hopin; Struik, Laura L. "A picture tells a thousand words: a content analysis of concussion-related images online", Published in Physical Therapy in Sport Vol. 21, Issue September 2016, p. 82-86. (2016)

Available from: http://dx.doi.org/10.1016/j.ptsp.2016.03.001

(C) 2016 This is an open access article under the CC BY-NC-ND license (http://creativecommons.org/licenses/by-nc-nd/4.0/).

Accessed from: http://hdl.handle.net/1959.13/1343361 
Original research

\section{A picture tells a thousand words: A content analysis of concussion-related images online}

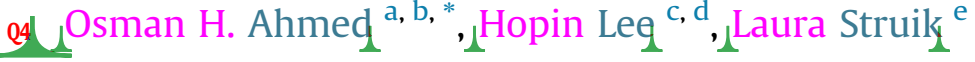

${ }^{a}$ Faculty of Health and Social Sciences, Bournemouth University, Bournemouth, UK

${ }^{\mathrm{b}}$ The FA Centre for Disability Football Research, St Georges Park, Burton-Upon-Trent, UK

${ }^{\mathrm{c}}$ Neuroscience Research Australia, Sydney, Australia

d Prince of Wales Clinical School, University of New South Wales, Sydney, Australia

e University of British Columbia, Okanagan Campus, Canada

University of British Columbia, Okanagan Campus, Canada

\section{A R T I C L E I N F O}

\section{Article history:}

Received 17 August 2015

Received in revised form

21 February 2016

Accepted 1 March 2016

Keywords:

Concussion

Social media

Knowledge translation

Public health

Q2 Society

A B S T R A C T

Background and aim: Recently image-sharing social media platforms have become a popular medium for sharing health-related images and associated information. However within the field of sports medicine, and more specifically sports related concussion, the content of images and meta-data shared through these popular platforms have not been investigated. The aim of this study was to analyse the content of concussion-related images and its accompanying meta-data on image-sharing social media platforms. Methods: We retrieved 300 images from Pinterest, Instagram and Flickr by using a standardised search strategy. All images were screened and duplicate images were removed. We excluded images if they were: non-static images; illustrations; animations; or screenshots. The content and characteristics of each image was evaluated using a customised coding scheme to determine major content themes, and images were referenced to the current international concussion management guidelines.

Results: From 300 potentially relevant images, 176 images were included for analysis; 70 from Pinterest, 63 from Flickr, and 43 from Instagram. Most images were of another person or a scene (64\%), with the primary content depicting injured individuals (39\%). The primary purposes of the images were to share a concussion-related incident (33\%) and to dispense education (19\%). For those images where it could be evaluated, the majority (91\%) were found to reflect the Sports Concussion Assessment Tool 3 (SCAT3) guidelines.

Conclusions: The ability to rapidly disseminate rich information though photos, images, and infographics to a wide-reaching audience suggests that image-sharing social media platforms could be used as an effective communication tool for sports concussion. Public health strategies could direct educative content to targeted populations via the use of image-sharing platforms. Further research is required to understand how image-sharing platforms can be used to effectively relay evidence-based information to patients and sports medicine clinicians.
\end{abstract}

() 2016 Elsevier Ltd. All rights reserved.

\section{Introduction}

Information sharing and knowledge dissemination is an integral aspect of the management and prevention of many sporting injuries. In the field of sports concussion the importance of accurate, evidence-based knowledge dissemination was first highlighted at

\footnotetext{
* Corresponding author. Faculty of Health and Social Sciences, Bournemouth University, Royal London House, Christchurch Road, Bournemouth, Dorset, BH1 3LT, UK. Tel.: +44 (0)1202 524111.

E-mail address: osman.hassan.ahmed@gmail.com (O.H. Ahmed).
}

the 2008 International Conference on Concussion in Sport (McCrory et al., 2008; Provvidenza \& Johnston, 2009). These discussions have further developed and feature in the current consensus statement (McCrory et al., 2012), and from a medical and epidemiological stand-point sports concussion is a serious public health problem (Wiebe, Comstock, \& Nance, 2011). Recently there has been considerable focus and attention on sports concussion in the mainstream media, both at community (The Irish News, 2014) and elite (The New York Times, 2014) athletic levels. In parallel, extensive research has shown that social media platforms such as Facebook (Ahmed et al., 2010), Twitter (Sullivan et al., 2012), and YouTube (Williams et al., 2014) are being used by patients and 
healthcare professionals to share concussion-related information. The functionality of these social media technologies allied to the popularity of social media means that the general public's exchange of concussion information has never been greater.

Popularity and usage of social media technologies evolve rapidly. In recent years, there has been a significant shift from basic messaging platforms (e.g. Twitter) to more sophisticated image sharing platforms. According to the Pew Research Center's Internet project, $54 \%$ of internet users post original photos or videos to websites and $47 \%$ share photos or videos they found elsewhere online (The Pew Internet and American Life Project, 2013). In keeping with this, web and mobile platforms are being used to upload and share health-related images (O'Mara, 2012). Some of the fastest growing Social Networking Sites (SNSs) such as Pinterest, Flickr and Instagram are those that are dedicated to sharing images. Pinterest aims to connect people based on shared tastes and interests through images (Stellrecht, 2012) (called "pins"), and currently has 70 million registered users worldwide. Flickr is another popular image-sharing website created 10 years ago as an image-sharing community (Zeng \& Wei, 2013) and currently has over 87 million users (Statistica Social Networks, 2014). More recently, Instagram has emerged as a major image-sharing social media platform. Since its recent inception in 2010, it has already accumulated 300 million users (Statistica Social Networks, 2014). Instagram enables users to take pictures and videos, apply digital filters to them, and share them instantly on its own platform and also via other major social networking sites (including on Facebook and Twitter).

Although these image-sharing sites are growing in usage, there has been little exploration of their use in healthcare. Two studies have examined the use of Flickr related to health; Renner et al. (2012) described the use of images on Flickr in relation to perceived HIV risk, whilst Yom-Tov and colleagues (2012) outlined the differences between pro-anorexia and pro-recovery images on Flickr. Both studies used Flickr to locate user uploaded images as sources of information together with the surrounding meta-data, and YomTov et al. used a set of search terms to identify images that depicted an individual's orientation towards 'pro-anorexic' behaviour (YomTov et al., 2012). Health-related research conducted on Pinterest is minimal at present, with Shellenbarger and Robb (2013) providing a discussion of the use of Pinterest in enhancing active and engaged learning strategies for nursing education. Although these studies provide preliminary insight into the content of health-related information on image-sharing platforms, to the best of our knowledge there has been no studies conducted to analyse health-related images in the discipline of sports medicine.

At present it is not known what type of concussion-related information is being disseminated through image-sharing platforms and whether their content reflects best-practice concussion guidelines. Undertaking content analyses of online images related to sports-related concussion will provide an understanding of the type and quality of information that is disseminated through these emerging SNSs. The purpose of this study was to systematically identify and analyse concussion-related images shared on popular image-sharing sites with reference to the current international concussion guidelines.

\section{Methods}

We used a systematic review process informed by existing methodologies from previous studies that evaluated image-sharing websites (Renner et al., 2012; Yom-Tov et al., 2012). The multi-stage data extraction process and analysis are shown in Fig. 1. As this study did not involve direct contact with human participants,

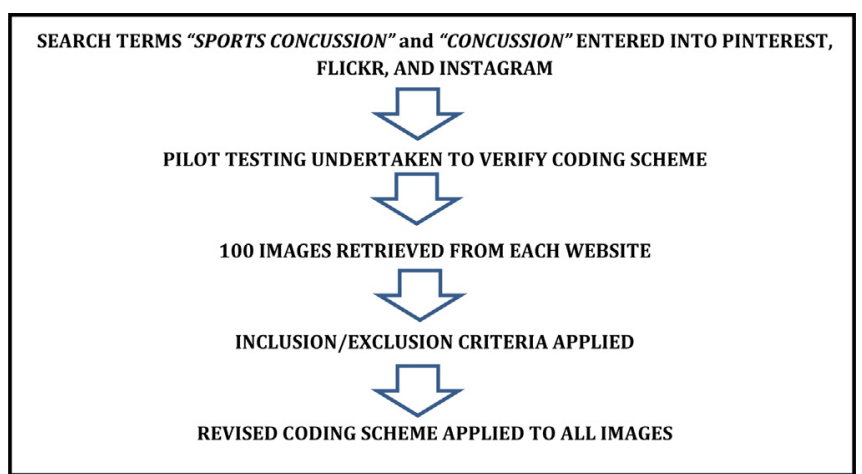

Fig. 1. Pathway involved in data extraction and analysis.

ethical approval was not sought. All of the data for this study was from freely available content on the internet.

\subsection{Sources of data}

The top three image-sharing platforms were selected based on their popularity (ranked by their total number of worldwide users), and these were Pinterest, Flickr and Instagram. For consistency and rigor, all searches were conducted on web-based versions of each of these websites (i.e. www.pinterest.com/, www.flickr.com/ and http://web.stagram.com/) using the web browser Google Chrome.

\subsubsection{Search strategy}

For each of the three websites (Pinterest, Flickr, and Instagram), the following search terms were used: "sports concussion" and "concussion". The first 50 images from each website were retrieved for analysis. All images were retrieved in the same 24-h window which ran from 10am GMT 12th August 2013 to 10am GMT 13th August 2013.

After retrieving all images, we removed duplicate images and applied the predefined inclusion/exclusion criteria. Images were excluded if they were: irrelevant photos/associated text (not related to sports related concussion, for example the music band "Concussion"); videos (i.e. non-images); illustrations, artistic/ computer-generated animation; screenshots/screencasts. For those cases where there were duplicate images, the first version of the image posted chronologically was included.

\subsubsection{Pilot testing and development of the data coding scheme}

A sample of 15 images was assessed prior to data collection/ analysis in order to develop and refine the coding scheme. Five images that were not retrieved from the 'main search' were taken from each of the three sites and each member of the research team (OHA/LS/HL) reviewed these images independently. By using a predeveloped coding scheme, the results of the pilot test were used to allow our research team to discuss how final coding decisions for each of the images was reached. Subsequently, minor alterations were made to the coding scheme before the main analysis. The outline and descriptions of the developed coding scheme are outlined in Table 1.

\subsubsection{Content analysis}

We used content analysis to evaluate the included images (Downe-Wamboldt, 1992), and this methodology has been used in similar studies that evaluated online concussion information (Ahmed et al., 2010; Sullivan et al., 2012). The categories in Table 1 were derived through an iterative process of discussion between the investigators to generate elements which would represent the

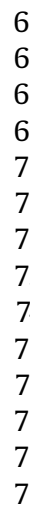


content of the images posted. The recent societal phenomenon of "selfies" (photographs individuals take of themselves) (BBC News, 2013b) indicated that a proportion of the images shared on these websites may be of this nature. To categorise this characteristic of each image, the coding aspect "type of image" was included. One of the key areas of interest was to identify why individuals were electing to share concussion images online. Following a series of discussions within the research team and examination of studies which have conducted similar concussion-related content analysis (Ahmed et al., 2010; Sullivan et al., 2012), categories were generated which attempted to capture this data (Table 1 ).

Best-practice management guidelines for concussion were created following the first International Conference on Concussion in Sport in 2001, and the most recent iteration of these was the Sports Concussion Assessment Tool 3 (SCAT3) which was generated in 2012 (McCrory et al., 2012). The SCAT3 (2013) contains bestpractice information relating to concussion management and therefore was used as a benchmark for the accuracy of the information depicted by the images. Although it was unrealistic to expect that an image could satisfy all of the information contained in the SCAT3, it was anticipated that an overall message would emerge from each picture that would either reflect the core principles of SCAT3 or be contrary to it. An example of an image supporting the SCAT3 guidelines would be where an individual posted an image in hospital indicating that they were having a consultation with a doctor following their injury, whilst an example of an image in contrary to the SCAT3 guidelines would be an individual sharing an image of them at a music concert the same night as their injury. In addition to the coding aspects related to the images, available metadata (surrounding data which is used to describe or provide commentary to a picture or image) associated with each image was also extracted (where available) in order to gain a comprehensive understanding of each image.

Images were independently coded by three of the investigators (OHA, LS, HL) and the results were tabulated. Secondary verification of each of the images was performed by a second member of the research team, and categories were established via discussion between each of the two investigators. In instances where two investigators did not reach agreement, a third investigator was included to reach consensus.

\section{Results}

Following the application of the inclusion and exclusion criteria, 176 images were included for analysis (see Fig. 2). Of these, 70 (40\%) were from Pinterest, 63 (36\%) were from Flickr, and 43 (24\%) were from Instagram. As shown in Table 2 the majority of included images were of another person(s) or scene (64\%), followed by infographics/non-photos (23\%) and "selfies" (13\%). The content of the images primarily consisted of injured individuals (39\%), with smaller proportion of images showing un-injured individuals (27\%), an object or product (11\%), a medical intervention taking place (4\%), or other content (19\%).

The primary purpose of the images was to share a concussionrelated incident which was seen in 33\% of all images. Lesser numbers of images were shown to dispense concussion education (19\%), display concussion-related advocacy or policies (12\%), advertise a product and display concussion management (11\% respectively), provide news related to concussions (9\%), or promote concussion-related research (5\%).

For most images in this study (66\%), it was not possible to determine the information quality i.e. whether the overall message from the image reflected or contradicted the SCAT3 concussion guidelines. Of those images where the information quality could be identified, 30\% were found to reflect the SCAT3 guidelines and 4\% were found to contradict the SCAT3 guidelines.

\section{Discussion}

This study adopted an innovative approach by taking a snapshot of how users of image-sharing SNSs are utilising these platforms in relation to sports concussion. Using multi-platform analysis for a specific sports injury ensured that a representative sample of images were retrieved from various platforms to allow a wider analysis than conducted in previous studies that used single platforms (Renner et al., 2012; Yom-Tov et al., 2012). While content analyses have been used to evaluate concussion-related information shared on other social media platforms (Ahmed et al., 2010; Sullivan et al.,

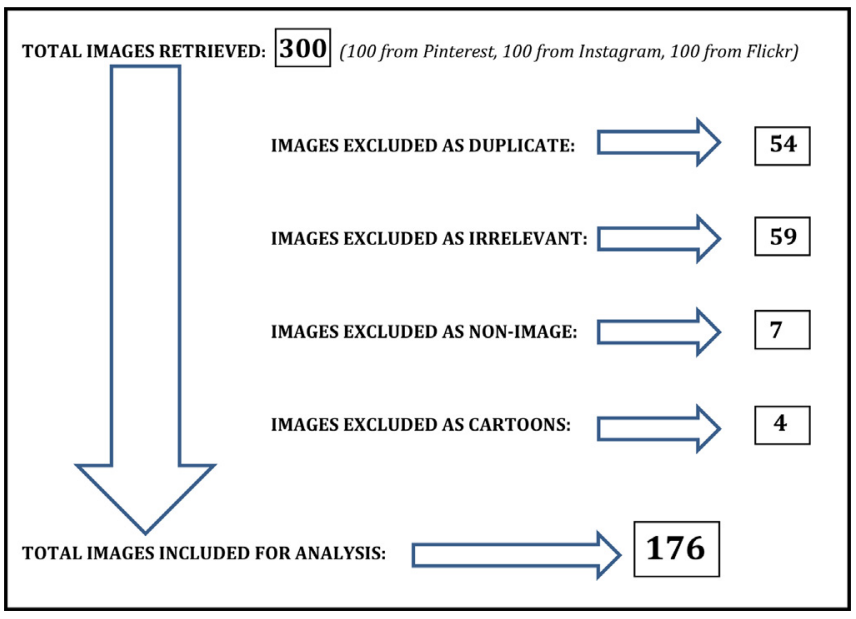

Fig. 2. Breakdown of excluded images.

Table 1

Coding scheme, rationale, and categories generated.

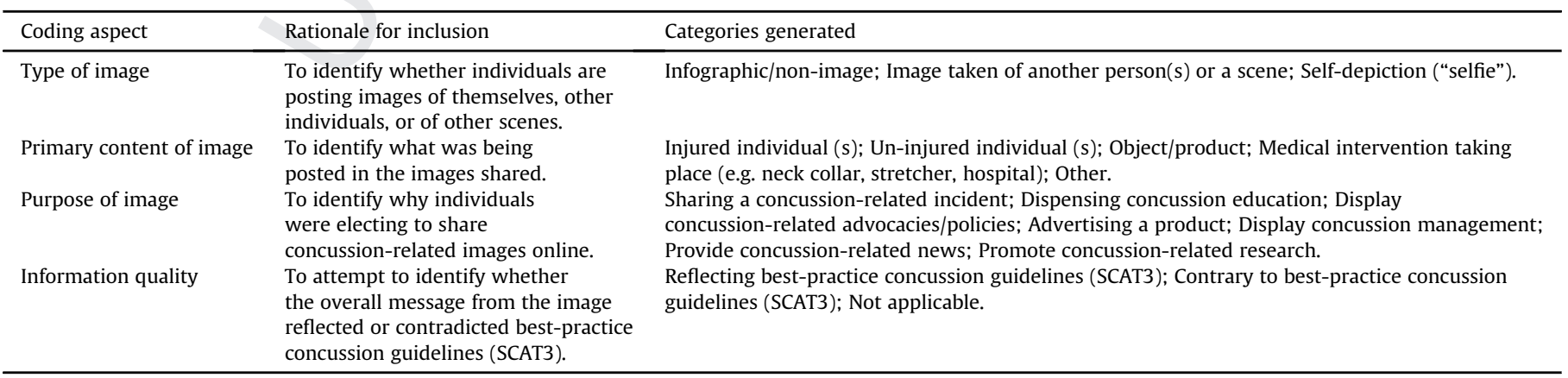


Table 2

Categorisation of images.

\begin{tabular}{|c|c|c|}
\hline Category & Number of images $(\mathrm{N})$ & Proportion of all images analysed (\%) \\
\hline \multicolumn{3}{|l|}{ Type of image } \\
\hline Infographic/non-photo & 41 & 23 \\
\hline Image taken of another person(s) or a scene & 113 & 64 \\
\hline Self-depiction ("selfie") & 22 & 13 \\
\hline \multicolumn{3}{|l|}{ Primary content of image } \\
\hline Injured individuals & 69 & 39 \\
\hline Un-injured individuals & 47 & 27 \\
\hline Object or product & 19 & 11 \\
\hline Medical intervention taking place & 8 & 4 \\
\hline Other & 33 & 19 \\
\hline \multicolumn{3}{|l|}{ Primary purpose of image } \\
\hline Share a concussion-related incident & 58 & 33 \\
\hline Dispense concussion education & 33 & 19 \\
\hline Display concussion-related advocacy or policies & 21 & 12 \\
\hline Advertise a product & 19 & 11 \\
\hline Display concussion management & 20 & 11 \\
\hline Provide concussion related news & 16 & 9 \\
\hline Promote concussion-related research & 9 & 5 \\
\hline \multicolumn{3}{|l|}{ Information quality } \\
\hline Adheres to the SCAT3 guidelines & 52 & 30 \\
\hline Contrary to the SCAT3 guidelines & 7 & 4 \\
\hline Unable to determine & 117 & 66 \\
\hline
\end{tabular}

2012; Williams et al., 2014), the functionality of each platform renders differences in the content that is shared. Evaluation on YouTube concussion content primarily shows individuals sustaining an injury (Williams et al., 2014); Twitter is mainly used to disseminate concussion news (Sullivan et al., 2012); and Facebook is used to foster peer support to individuals who had sustained a concussion (Ahmed et al., 2010). It is reasonable to infer from these differences that the form of online media platform influences the content that is disseminated and shared with the general public.

Of those images where the adherence to best practices was able to be determined $(n=59)$, it was encouraging that far more images reflected the best practice concussion management guidelines $(\mathrm{n}=52$ or $88 \%$ ) than were in contravention of the guidelines $(\mathrm{n}=7$ or $12 \%$ ). Despite concerns and limitations of using social media for health communication being reported in the literature (Moorhead et al., 2013), the overall quality of the information being conveyed via the retrieved images in this study indicates that image-sharing sites should be included as part of a comprehensive, holistic approach towards information dissemination strategies for concussion. Our findings support the recommendations of Provvidenza et al. (2013) who cited the use of social media as an innovative approach to assist knowledge translation in concussion. Given the unique role that images play in presenting information without text, it provides an opportunity to bring health information to audiences with special or different needs (Adams, 2010). Prior research has shown that concussion information online is often at a standard where it is not comprehensible for the general population (Ahmed et al., 2012), and harnessing platforms such as Pinterest "pinboards" may serve as an educational resource for individuals with low literacy and enable concussion information to be shared regardless of reading ability or comprehension level.

The most common types of images retrieved in this study were of another person or scene (64\%) and infographics or non-photos (23\%). The infographics retrieved for this study often contained concussion-related information which had been presented in a format suitable for the image-sharing site, and as such they could constitute a means of promoting concussion awareness and education. This finding is encouraging in light of previous work indicating that real-life images are effective in conveying health information (Houts et al., 1998, 2006) and also that infographics are popular visual communication tools for relaying complex health information (Smiciklas, 2012). In a recent content analysis of Chronic Obstructive Pulmonary Disease-related Pinterest images, the authors found that images of real people and infographics depicting self-management information received the greatest amount of user engagement though user comments and "likes" (Paige et al., 2015).

In our study the proportion of "selfies" was low, with only $13 \%$ being photographs that individuals were taking of themselves. The role of selfies in modern society is notable (BBC News, 2013a), and websites such as "Facing Concussion" (2014) are dedicated to sharing selfies of individuals with concussion. Monitoring the content and metadata of "selfies" shared by individuals with sports concussion may enable further understanding of the psychosocial aspects of sports concussion. With much of the information being shared by individuals in this manner, it is noteworthy that there are quality standards in use for health-related websites (The Health on the Net Foundation, 2014) and for smartphone apps (US Food and Drug Administration, 2013) but at present there are no specific guidelines or regulations regarding the posting of health-related images online. The examples of static images (and surrounding metadata) seen in this study may represent a source of novel and potentially valuable information, both for improving the understanding of sports concussion but more importantly as a potential dissemination technique for concussion education and awareness. The increasing use and popularity of image-sharing sites would indicate that major public health organisations and sports medicine clinicians could consider the use of image-sharing sites for information dissemination for sports concussion.

Given that there was very limited prior work and literature to draw upon in this area, several limitations are identified. Attributing categories to images was not always straightforward, and this was especially pertinent when attempting to identify if the images were in keeping with the SCAT3. The proportion of images (66\%) where information quality was not able to be determined highlights this. Differences between each of the communities in terms of their aims and target audiences are also acknowledged. The majority of Flickr users are photography hobbyists (from amateurs to professionals (The Pew Internet and American Life Project, 2013)), whereas Instagram users are younger and primarily use the site to share photos with their friends (Marketing Charts, 2013). These characteristics may have accounted for differences between 
the sites, however as there was no direct interaction with the individuals posting these images, cross-platform differences remain speculative. It is accepted that this study was non-exhaustive and involved a sample of images shared on a finite number of imagesharing sites (this approach being adopted in previous social media concussion research (Ahmed et al., 2010; Sullivan et al., 2012)), and therefore the generalizability of the sampled images is limited.

Several future areas of research are warranted. One key area is the dialogue that surrounds these online images; Flickr and Instagram both allow users to send private messages to each other, but given that this interaction is not public it was not possible to measure this in our study. Discussion boards and comment threads on Flickr are accessible however, and may constitute an area of exploration for concussion-related dialogue. In addition, many of the images retrieved in this study used associating hashtags (identifying tags) for identification and labelling purposes. It is possible that sentiments regarding injury and frustration about return to play issues might be depicted in such dialogue. Keywords such as "\#allgood" were observed, with these colloquial hashtags potentially downplaying the seriousness of a concussion. There was no formal evaluation of this, and future studies should explore the relationships between images and accompanying dialogue. Consultation with individuals sharing concussion-related images online is also indicated, as a better understanding of the intention of users posting images on these sites may allow health organisations (including sports medicine associations) to interact via imagesharing sites to achieve a public health benefit.

\section{Conclusion}

The ability to rapidly disseminate rich information though photos, images, and infographics to a wide-reaching audience suggests that image-sharing social media platforms could be used as an effective communication tool for sports concussion. Public health strategies could direct educative content to targeted populations via the use of image-sharing platforms, and clinicians should be aware their athletes under their care may be using these sites to share their concussion experiences. Further research is required to understand how image-sharing platforms can be used to effectively relay evidence-based information to patients and sports medicine clinicians.

\section{Conflict of interest}

None declared.

Ethical approval

None declared.

\section{Funding}

None declared.

\section{Acknowledgements}

None declared.

\section{References}

Adams, S. A. (2010). Revisiting the online health information reliability debate in the wake of "web 2.0": an inter-disciplinary literature and website review. International Journal of Medical Informatics, 79, 391-400.

Ahmed, O. H., Sullivan, S. J., Schneiders, A. G., et al. (2010). iSupport: do social networking sites have a role to play in concussion awareness? Disability \& Rehabilitation, 32, 1877-1883.

Ahmed, O. H., Sullivan, S. J., Schneiders, A. G., et al. (2012). Concussion information online: evaluation of information quality, content and readability of concussion-related websites. British Journal of Sports Medicine, 46, 675-683.
Downe-Wamboldt, B. (1992). Content analysis: method, applications, and issues. Health Care for Women International, 13, 313-321.

Facing Concussion. (2014). Available from http://www.facingconcussion.com/site/ Accessed 12.07.2014.

Houts, P. S., Bachrach, R., Witmer, J. T., et al. (1998). Using pictographs to enhance recall of spoken medical instructions. Patient Education and Counselling, 35 83-88.

Houts, P. S., Doak, C. C., Doak, L. G., et al. (2006). The role of pictures in improving health communication: a review of research on attention, comprehension, recall, and adherence. Patient Education and Counselling, 61, 173-190.

Marketing Charts. (29th October 2013). The demographics of instagram and snapchat users. Available from: http://www.marketingcharts.com/wp/online/thedemographics-of-instagram-and-snapchat-users-37745/ Accessed 6.07.14.

McCrory, P., Meeuwisse, W. H., Aubry, M., et al. (November 2012). Consensus statement on concussion in sport: the 4th International conference on concussion in sport held in Zurich. British Journal of Sports Medicine, 2013(47), 250-258.

McCrory, P., Meeuwisse, W., Johnston, K. M., et al. (November 2008). Consensus statement on concussion in sport: the 3rd international conference on concussion in sport held in Zurich. British Journal of Sports Medicine, 2009(43), i76-90.

Moorhead, S. A., Hazlett, D. E., Harrison, L., et al. (2013). A new dimension of health care: systematic review of the uses, benefits, and limitations of social media for health communication. Journal of Medical Internet Research, 15, e85.

BBC News. (19th November 2013). 'Selfie' named by Oxford Dictionaries as word of 2013. Available from: http://www.bbc.co.uk/news/uk-24992393 Accessed 12.07.14

BBC News. (7th June 2013). Self-portraits and social media: the rise of the 'selfie'. 2013. Available from: http://www.bbc.co.uk/news/magazine-22511650 Accessed 17.04.14.

O'Mara, B. (2012). Social media, digital video and health promotion in a culturally and linguistically diverse Australia. Health Promotion International, 28, 466-476.

Paige, S. R., Stellefson, M., Chaney, B. H., et al. (2015). Pinterest as a resource for health information on chronic obstructive pulmonary disease (COPD): a social media content analysis. American Journal of Health Education, 46, 241-251.

Provvidenza, C., Engebretsen, L., Tator, C., et al. (2013). From consensus to action: knowledge transfer, education and influencing policy on sports concussion. British Journal of Sports Medicine, 47, 332-338.

Provvidenza, C. F., \& Johnston, K. M. (2009). Knowledge transfer principles as applied to sport concussion education. British Journal of Sports Medicine, 43, i68-i75.

Renner, B., Schmälzle, R., \& Schupp, H. (2012). First impressions of HIV risk: it takes only milliseconds to scan a stranger. PLoS One, (1), 7.

SCAT3. (2013). British Journal of Sports Medicine, 47, 259-262.

Shellenbarger, T., \& Robb, M. (2013). Pinstructive ideas: using a social networking bulletin board for nursing education. Nurse Educator, 38, 206-209.

Smiciklas, M. (2012). Infographics: Using pictures to communicate and connect with your audiences. Indianapolis, IN: Pearson Education, Inc.

Statistica Social Networks: global sites ranked by users 2014. Available from:: http://www.statista.com/statistics/272014/global-social-networks-ranked-bynumber-of-users/. Accessed 26.12.14.

Stellrecht, E. (2012). Pinterest interest: converting a consumer health guide Wiki into a Pinterest page. Journal of Consumer Health on the Internet, 16, 403-408.

Sullivan, S. J., Schneiders, A. G., Cheang, C. W., et al. (2012). What's happening? a content analysis of concussion-related traffic on Twitter. British Journal of Sports Medicine, 46, 258-263.

The Health on the Net Foundation. (2014). Available from: http://www.hon.ch/ HONcode/ Accessed 17.07.14.

The Irish News. (1st May 2014). Campaign highlights dangers of concussion. Available from: http://www.irishnews.com/sport/campaign-highlights-dangers-ofconcussion-1351906 Accessed 5.05.14.

The New York Times. (7th April 2014). Europe, echoes of America as concussions spur debate. Available from: http://www.nytimes.com/2014/04/06/sports/in-europeechoes-of-america-as-concussions-spur-debate.html?_r=0 Accessed 10.04.14.

The Pew Internet and American Life Project. (28th October 2013). Photo and video sharing grow online. Available from: http://www.pewinternet.org/Reports/2013/ Photos-and-videos.aspx Accessed 16.01.14.

US Food and Drug Administration. (23rd September 2013). Available from: http:/ www.fda.gov/newsevents/newsroom/pressannouncements/ucm369431.htm Accessed 12.07.14

Wiebe, D. J., Comstock, R., \& Nance, M. L. (2011). Concussion research: a public health priority. Injury Prevention, 17, 69-70.

Williams, D., Sullivan, S. J., Schneiders, A. G., et al. (2014). Big hits on the small screen: an evaluation of concussion-related videos on YouTube. British Journal of Sports Medicine, 48, 107-111.

Yom-Tov, E., Fernandez-Luque, L., Weber, I., et al. (2012). Pro-anorexia and prorecovery photo sharing: a tale of two warring tribes. Journal of Medical Internet Research, 14, e151.

Zeng, X., \& Wei, L. (2013). Social ties and user content generation: evidence from Flickr. Information Systems Research, 24, 71-87. 